\title{
Properties of melanin from black tea leaves
}

\author{
Yu Zou*, Yue Zhao \\ College of Life Science, Dalian Nationalities University, 18 Liaohe West Road, Dalian Development Area, \\ 116600 Dalian, Liaoning, China
}

*Corresponding author, e-mail: yuzou126@126.com

Received 8 May 2015

Accepted 1 Nov 2015

\begin{abstract}
In this study, partial physicochemical properties and antioxidant activities of melanin from black tea leaves were studied. The results show that black tea melanin powder was dark tinted red and yellow $\left(L^{*}=36.47, a^{*}=0.33\right.$, $\left.b^{*}=0.17\right)$. Black tea melanin solution exhibited strong optical absorbance in a wide UV-Vis spectral range and was relatively stable in the range of $25-100^{\circ} \mathrm{C}$ and under natural light. Furthermore, black tea melanin exhibited a strong superoxide- and hydroxyl-radical scavenging activity. Results of the present study indicated that black tea melanin could be potentially used as a food colourant with health benefits.
\end{abstract}

KEYWORDS: antioxidant activity, stability, food colourant

\section{INTRODUCTION}

The harmful effects to human health of some synthetic pigments have led to public interest in natural pigments as an alternative in the food industry. Melanin is a high molecular weight pigment (generally of red, black, or brown colour) produced from oxidative polymerization of phenolic or indolic compounds ${ }^{1}$. Melanin from natural sources, such as black-bone silky fowl ${ }^{2}$, Osmanthus fragrans seeds ${ }^{3}$, Auricularia auricula fruit-bodies ${ }^{4}$, and $\mathrm{Hy}$ poxylon archeri ${ }^{5}$ of microorganisms possess similar physicochemical properties. These physicochemical properties include strong light absorbance, unusual solubility, and remarkable redox properties. In addition, melanin has a number of healthful functions, such as antioxidant ${ }^{6,7}$ and anti-HIV activity ${ }^{8,9}$. As a result, consumers are demanding to use melanin in the food industry as a safe natural colourant rather than a synthetic dye.

Black tea is a traditional and famous drink, rich in melanin, and is increasingly popular as a black food in China. Melanin is considered to be one of the most important functional component of black food. In a previous study, melanin was successfully extracted from black tea leaves and some physicochemical properties including solubility and redox properties were described ${ }^{10}$. However, up to now, no further investigation has been conducted on other physicochemical properties and biological activities of melanin from black tea leaves.

In this study, melanin was isolated and purified from black tea leaves and its partial physicochemical properties were investigated. Meanwhile, scavenging activity of black tea melanin against free radicals was assessed in seeking new biological activities to be used as proof-of-principle in the food industry.

\section{MATERIALS AND METHODS}

\section{Materials and reagents}

Black tea leaves were purchased from a local market in Dalian City (Liaoning Province, China), pulverized and sifted through a 60-mesh sieve. The powder was stored in dark bags to be kept from moisture and light. Butylated hydroxytoluene (BHT) was purchased from Sigma Chemicals Co. (St. Louis, MO, USA). All the other chemicals and reagents used in the experiment were of analytical grade.

\section{Extraction and purification of melanin}

The extraction process of melanin was carried out according to the method of Zou et al ${ }^{11}$ with modification. About $2 \mathrm{~g}$ of black tea leaf powder was washed with running water at a ratio of $60 \mathrm{ml} / \mathrm{g}$ (water/raw materials) for $5 \mathrm{~min}$, followed by centrifugation at $2100 \mathrm{~g}$ for $5 \mathrm{~min}$. The precipitate was immersed into water and the initial $\mathrm{pH}$ was adjusted to 12 with $1 \mathrm{M} \mathrm{NaOH}$. Then the mixture was put into a conical flask for incubation extraction at $100^{\circ} \mathrm{C}$ for $40 \mathrm{~min}$. After that the sample was centrifuged at $2100 \mathrm{~g}$ for $5 \mathrm{~min}$ and the supernatant containing melanin was obtained and stored at $4{ }^{\circ} \mathrm{C}$ in the dark. Purification of melanin was performed as described by Wu et $\mathrm{al}^{5}$ with modification. Melanin extract was first adjusted to $\mathrm{pH} 2.0$ with $3 \mathrm{M} \mathrm{HCl}$ to precipitate 
melanin, followed by centrifugation at $13000 \mathrm{~g}$ for $20 \mathrm{~min}$ and the pellet was collected. The pellet was washed three times with chloroform, ethyl acetate, and ethanol. Finally, the purified melanin was lyophilized and stored at $-20^{\circ} \mathrm{C}$ until further use. To avoid changing the melanin properties, samples were stored for no longer than 10 days.

\section{Visual colour and UV-Vis absorption spectrum of melanin}

Visual colour of melanin powder was measured using a Minolta colorimeter (CR-400, Minolta Camera Co. Ltd., Osaka, Japan) with the Hunter Lab colour system. The colour values were expressed as $L^{*}$ (whiteness or brightness/darkness), $a^{*}$ (redness/ greenness), and $b^{*}$ (yellowness/blueness). Melanin was dissolved in alkaline distilled water $(\mathrm{pH} 9.0)$ at a final concentration of $50 \mathrm{mg} / \mathrm{l}$. The UV-Vis absorption spectrum of the melanin solution was scanned using a UV-2802 diode array spectrophotometer (Unico Instrument Co. Ltd., Princeton, NJ, USA) at wavelengths ranging from $200-800 \mathrm{~nm}$.

\section{Effects of temperature and light on melanin stability}

The thermal stability of melanin solution was measured after treatment in a thermostatically controlled bath at $25,50,75$, and $100^{\circ} \mathrm{C}$. The samples were held at each temperature for specific times and then cooled immediately in an ice bath. Subsequently, the absorbance of the solutions was recorded at $400 \mathrm{~nm}$ to attain the heat stability of melanin ${ }^{7}$. In addition, melanin solution was held under natural light or at dark place for specific time and the absorbance was determined at $400 \mathrm{~nm}$ to determine the melanin light stability.

\section{Superoxide radical scavenging activity of melanin}

The superoxide radical scavenging activities of black tea melanin and BHT were evaluated according to the method detailed by Martinez et al ${ }^{12}$. Each $3 \mathrm{ml}$ of reaction mixture contained $50 \mathrm{mM}$ of $\mathrm{Na}_{3} \mathrm{PO}_{4}$ buffer ( $\mathrm{pH}$ 7.8), $13 \mathrm{mM}$ of methionine, $2 \mu \mathrm{M}$ of riboflavin, $100 \mu \mathrm{M}$ of EDTA, $75 \mu \mathrm{M}$ of nitroblue tetrazolium (NBT), and $1 \mathrm{ml}$ of melanin or BHT of different concentrations $(0.2,0.4,0.6,0.8$, and $1.0 \mathrm{mg} / \mathrm{ml}$ ). The production of blue formazan was then followed by monitoring the increase in absorbance at $560 \mathrm{~nm}$ after a $10 \mathrm{~min}$ illumination from a fluorescent lamp. The entire reaction assembly was enclosed in a box lined with aluminium foil. Identical tubes with reaction mixture were kept in the dark and served as blanks. The scavenging activity of superoxide radical was calculated as $\left(A_{\mathrm{C}}-A_{\mathrm{S}}\right) / A_{\mathrm{C}}$, where $A_{\mathrm{C}}$ was the absorbance of the control (deionized water, instead of sample), and $A_{\mathrm{S}}$ was the absorbance of the test sample mixed with reaction solution.

\section{Hydroxyl radical scavenging activity of melanin}

The scavenging abilities of black tea melanin and BHT on hydroxyl radical were determined by the method previously reported by Sun and Kennedy ${ }^{13}$. Reaction mixtures in a final volume of $1.0 \mathrm{ml}$ contained deoxyribose $(60 \mathrm{mM})$, phosphate buffer (pH 7.4, $20 \mathrm{mM})$, ferric trichloride $(100 \mu \mathrm{M})$, EDTA $(100 \mu \mathrm{M}), \mathrm{H}_{2} \mathrm{O}_{2}(1 \mathrm{mM})$, and different concentrations of melanin or BHT $(0.2,0.4,0.6,0.8$, and $1.0 \mathrm{mg} / \mathrm{ml}$ ). The reaction solution was incubated for $1 \mathrm{~h}$ at $37^{\circ} \mathrm{C}$, and then $1.0 \mathrm{ml}$ of $1 \%$ thiobarbituric acid (TBA) and $1.0 \mathrm{ml}$ of $20 \%(\mathrm{v} / \mathrm{v}) \mathrm{HCl}$ were added to the mixture. The mixture was boiled for $15 \mathrm{~min}$ and cooled on ice. The absorbance of the resulting mixture was measured at $532 \mathrm{~nm}$. The scavenging activity of hydroxyl radical was calculated as $\left(A_{\mathrm{B}}-A_{\mathrm{S}}\right) / A_{\mathrm{B}}$, where $A_{\mathrm{B}}$ was the absorbance of the control (deionized water, instead of sample), and $A_{\mathrm{S}}$ was the absorbance of the test sample mixed with reaction solution.

\section{Statistical analysis}

The experimental results obtained were expressed as means \pm SD of triplicates. Statistical analysis was performed using Fisher's $F$-test and $p<0.05$ was regarded as significant.

\section{RESULTS AND DISCUSSION}

\section{Visual colour and UV-Vis absorption spectrum}

Results from the colorimeter indicated that black tea melanin powder presented lower $L^{*}$ value (36.47 \pm 0.29$), a^{*}$ value $(0.33 \pm 0.03)$, and $b^{*}$ value $(0.173 \pm 0.01)$ in Hunter Lab colour system. This indicates that black tea melanin powder was dark with a little red and yellow colour. The UV-Vis spectrum $(200-800 \mathrm{~nm})$ of black tea melanin is shown in Fig. 1. Melanin absorbed strongly in the UV region and progressively less as the wavelength increased. According to a previous report ${ }^{1}$, strong optical absorbance in a wide spectral range was one of the most conspicuous properties of melanin due to the high degree of conjugation in the molecule. Some melanins extracted from plant and animal, such as $O$. fragrans seeds ${ }^{3}$ and black-bone silky 


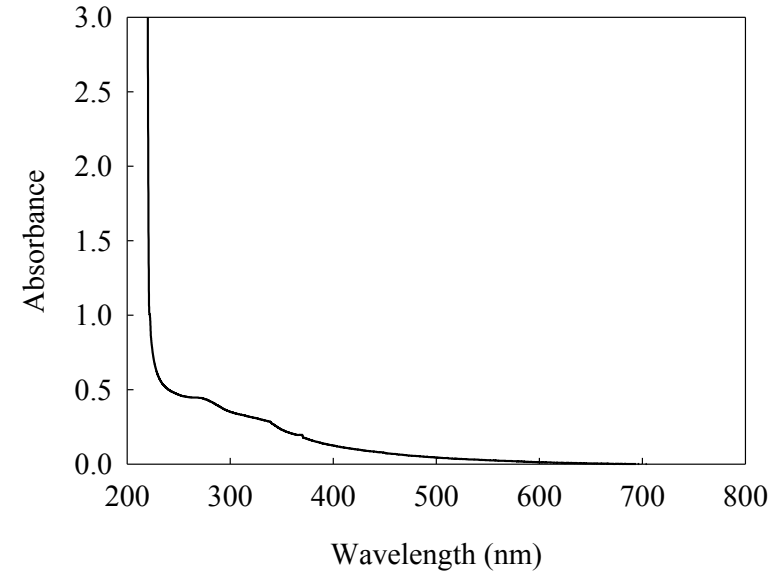

Fig. 1 UV-Vis spectrum of black tea melanin.

fow $1^{7}$, exhibited an additional shoulder at wavelengths $260-280 \mathrm{~nm}$, property which was similar to that of the melanin extracted from black tea leaves. It is well known that normal proteins had an absorption maximum at about $280 \mathrm{~nm}$. Thus black tea melanin might contain a certain amount of protein.

\section{Heat and light stabilities}

The effect of temperature on black tea melanin stability was determined to ascertain the potential use of the melanin as a natural colourant. The brown colour of the melanin was almost unchanged during heating. As shown in Table 1, the melanin loss was only $6 \%$ when the solution was heated at $100^{\circ} \mathrm{C}$ for $4 \mathrm{~h}$, indicating that melanin is thermally stable. Table 2 shows that the absorbance of black tea melanin solution was slightly declined under natural illumination. After being treated for 5 days, the absorbance of the melanin decreased slightly with a loss of 0.011, while the colour of the melanin solution had no obvious change indicating that light had less effect on melanin. This was in agreement with the results reported by Wang et $\mathrm{al}^{3}$.

\section{Superoxide radical scavenging activity}

Although superoxide was a relatively weak oxidant, its combination with other reactive species, such as nitric oxide and hydroxyl, might yield stronger reactive species which possessed greater oxidative ability than the precursor to initiate lipids peroxidation $^{14,15}$. Furthermore, superoxide is also known to indirectly induce lipid peroxidation as a result of $\mathrm{H}_{2} \mathrm{O}_{2}$ formation, creating precursors of hydroxyl radicals ${ }^{13}$. The hydrogen-donating ability is a primary index of antioxidants that donate hydrogen to superoxide radicals, resulting in detoxification and inhibition of the propagation phase of lipid oxidation. The results of the scavenging superoxide radical of black tea melanin and BHT are shown in Fig. 2. The scavenging activities of black tea melanin and BHT increased when the corresponding concentration increased. In the concentration range of $0.2-1.0 \mathrm{mg} / \mathrm{ml}$, black tea melanin possessed higher $(p<0.05)$ superoxide radical scavenging activity than BHT in a concentration-dependent manner. At an identical concentration, scavenge activity of black tea melanin on superoxide radical was stronger than melanin from black-bone silky fowl and synthetic melanin ${ }^{7}$.

\section{Hydroxyl radical scavenging activity}

Among the reactive oxygen radicals, hydroxyl radical is known as the most powerful radical. It could induce severe damage to adjacent biomolecules in

Table 1 Heat stability of black tea melanin.

\begin{tabular}{lccccr}
\hline $\begin{array}{l}\text { Temperature } \\
\left({ }^{\circ} \mathrm{C}\right)\end{array}$ & $0 \mathrm{~h}$ & $1 \mathrm{~h}$ & $2 \mathrm{~h}$ & $3 \mathrm{~h}$ & $4 \mathrm{~h}$ \\
\cline { 2 - 6 } & $0.525 \pm 0.003$ & $0.524 \pm 0.004$ & $0.523 \pm 0.003$ & $0.521 \pm 0.004$ & $0.519 \pm 0.001$ \\
\hline 25 & $0.525 \pm 0.003$ & $0.523 \pm 0.005$ & $0.518 \pm 0.003$ & $0.512 \pm 0.001$ & $0.508 \pm 0.003$ \\
50 & $0.525 \pm 0.003$ & $0.523 \pm 0.002$ & $0.516 \pm 0.001$ & $0.508 \pm 0.005$ & $0.501 \pm 0.002$ \\
75 & $0.525 \pm 0.003$ & $0.521 \pm 0.006$ & $0.512 \pm 0.004$ & $0.503 \pm 0.004$ & $0.494 \pm 0.002$ \\
100 & &
\end{tabular}

Table 2 Light stability of black tea melanin.

\begin{tabular}{lccrr}
\hline Light source & \multicolumn{4}{c}{ Absorbance } \\
\cline { 2 - 5 } & $0 \mathrm{~d}$ & $1 \mathrm{~d}$ & $3 \mathrm{~d}$ & $5 \mathrm{~d}$ \\
\hline Dark & $0.617 \pm 0.005$ & $0.617 \pm 0.005$ & $0.617 \pm 0.003$ & $0.615 \pm 0.002$ \\
Natural light & $0.617 \pm 0.005$ & $0.616 \pm 0.003$ & $0.612 \pm 0.004$ & $0.606 \pm 0.004$ \\
\hline
\end{tabular}




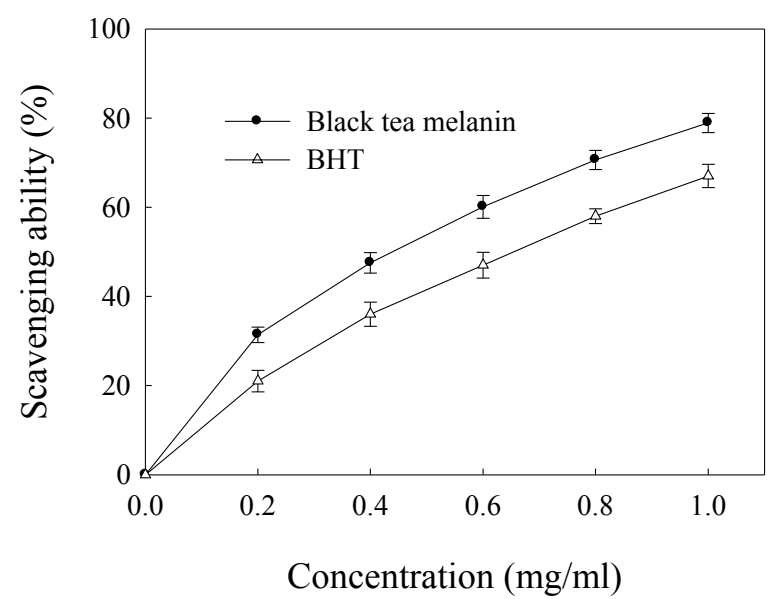

Fig. 2 Scavenging effect of black tea melanin on superoxide radicals.

the body, which result in cell damage that caused ageing, cancer, and several other diseases ${ }^{16}$. The removal of hydroxyl radical is probably one of the most effective ways to defend the body oxidative damage. Hydroxyl radical scavenging activity is therefore considered to be one of the most important antioxidant mechanisms. The scavenging abilities of black tea melanin and BHT on hydroxyl radical are shown in Fig. 3. With the increase of concentration, the scavenging activities of black tea melanin and BHT on hydroxyl radical also increased. However, the scavenging activities of black tea melanin increased from $37 \%$ up to $79 \%$, which was significantly higher $(p<0.05)$ than BHT. These results suggested that black tea melanin was better natural antioxidant than BHT in scavenging hydroxyl radical.

In conclusion, black tea melanin was prepared and its partial physicochemical properties and antioxidant activities were investigated. Melanin powder presented lower $L^{*}$ value (36.47), $a^{*}$ value $(0.33)$, and $b^{*}$ value (0.17) in visual colour. Melanin solution exhibited strong optical absorbance in a wide UV-Vis spectral range. It was relatively stable to temperature and light. In addition, the antioxidant activities of black tea melanin were higher than that of BHT. Black tea melanin could be further used as a natural food colourant.

Acknowledgements: This work was supported by the Doctor Research Fund of Dalian Nationalities University (0701-110015) and the Undergraduate Training Programs for Innovation and Entrepreneurship of Dalian Nationalities University (X201503051).

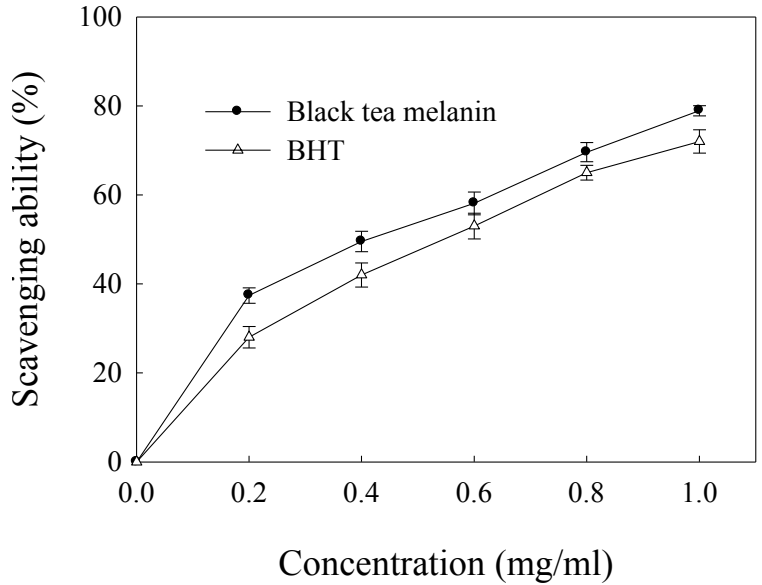

Fig. 3 Scavenging effect of black tea melanin on hydroxyl radicals.

\section{REFERENCES}

1. Riley PA (1997) Melanin. Int J Biochem Cell Biol 29, 1235-9.

2. Chen SR, Jiang B, Zheng JX, Xu GY, Li JY, Yang $\mathrm{N}$ (2008) Isolation and characterization of natural melanin derived from silky fowl (Gallus gallus domesticus Brisson). Food Chem 111, 745-9.

3. Wang H, Pan Y, Tang X, Huang Z (2006) Isolation and characterization of melanin from Osmanthus fragrans' seeds. LWT Food Sci Tech 39, 496-502.

4. Zou Y, Yang Y, Zeng B, Gu Z, Han Y (2013) Comparison of physicochemical properties and antioxidant activities of melanins from fruit-bodies and fermentation broths of Auricularia auricula. Int J Food Prop 16, 803-13.

5. Wu Y, Shan L, Yang S, Ma A (2008) Identification and antioxidant activity of melanin isolated from Hypoxylon archeri, a companion fungus of Tremella fuciformis. $J$ Basic Microbiol 48, 217-21.

6. Chiarelli-Neto O, Pavani C, Ferreira AS, Uchoa AF, Severino D, Baptista MS (2011) Generation and suppression of singlet oxygen in hair by photosensitization of melanin. Free Radic Biol Med 51, 1195-202.

7. Tu Y, Sun Y, Tian Y, Xie M, Chen J (2009) Physicochemical characterisation and antioxidant activity of melanin from the muscles of Taihe Black-bone silky fowl (Gallus gallus domesticus Brisson). Food Chem 114, 1345-50.

8. Manning JT, Bundred PE, Henzi P (2003) Melanin and HIV in sub-Saharan Africa. $J$ Theor Biol 223, 131-3.

9. Montefiori DC, Zhou JY (1991) Selective antiviral activity of synthetic soluble L-tyrosine and L-dopa melanins against human immunodeficiency virus in vitro. Antivir Res 15, 11-25.

10. Zou Y, Ma K, Tian M (2014) Physicochemical prop- 
erties and stability of melanin from black tea leaves. $J$ Chem Pharmaceut Res 6(7), 1560-2.

11. Zou Y, Xie C, Fang G, Gu Z, Han Y (2010) Optimization of ultrasound-assisted extraction of melanin from Auricularia auricula fruit bodies. Innovat Food Sci Emerg Tech 11, 611-5.

12. Martinez CA, Loureiro ME, Oliva MA, Maestri M (2001) Differential responses of superoxide dismutase in freezing resistant Solanum curtilobum and freezing sensitive Solanum tuberosum subjected to oxidative and water stress. Plant Sci 160, 505-15.

13. Sun YX, Kennedy JF (2010) Antioxidant activities of different polysaccharide conjugates (CRPs) isolated from the fruiting bodies of Chroogomphis rutilus (Schaeff.: Fr.) O. K. Miller. Carbohydr Polymer 82, 510-4.

14. Miguel MG, Nunes S, Dandlen SA, Cavaco AM, Antunes MD (2014) Phenols, flavonoids and antioxidant activity of aqueous and methanolic extracts of propolis (Apis mellifera L.) from Algarve, South Portugal. Food Sci Tech (Campinas) 34, 16-23.

15. Peksel A, Arisan-Atac I, Yanardag R (2010) Evaluation of antioxidant and antiacetylcholinesterase activities of the extracts of Pistacia atlantica desf. leaves. J Food Biochem 34, 451-76.

16. Yang X, Yan F, Huang S, Fu C (2014) Antioxidant activities of fractions from longan pericarps. Food Sci Tech (Campinas) 34, 341-5. 